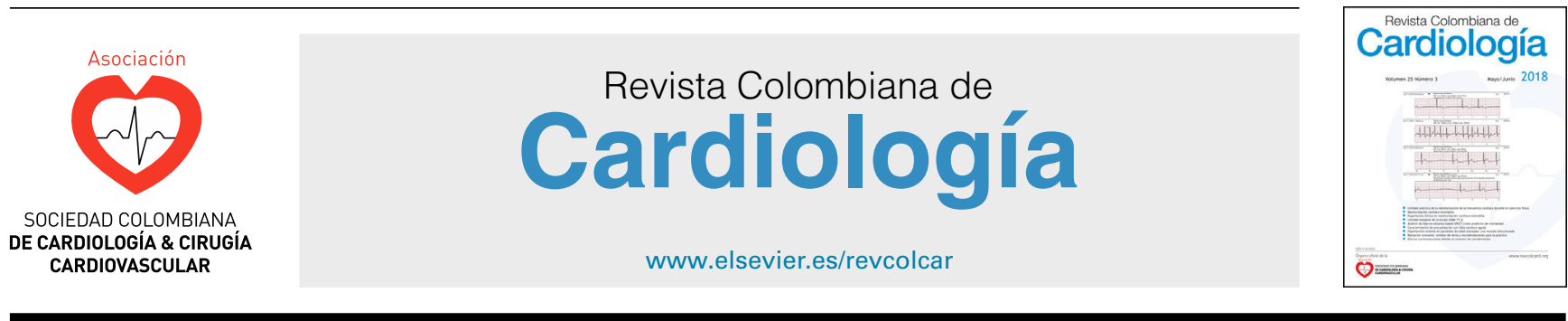

CARDIOLOGÍA DEL ADULTO - PRESENTACIÓN DE CASOS

\title{
Infarto de miocardio tras la administración de adrenalina intragingival ¿Una nueva causa de infarto agudo de miocardio sin enfermedad coronaria aterosclerótica obstructiva?
}

\author{
Pedro Pérez Díaz*, Jorge Martínez del Río, Raquel Frías García, \\ Alfonso Morón Alguacil y Alfonso Jurado Román
}

Departamento de Cardiología, Hospital General Universitario de Ciudad Real, Ciudad Real, España

Recibido el 3 de julio de 2019; aceptado el 21 de diciembre de 2019

Disponible en Internet el 31 de mayo de 2020

PALABRAS CLAVE

Cardiopatía

isquémica;

Infarto de miocardio;

Trombosis.
Resumen Es frecuente la relación entre la adrenalina administrada por vía intramuscular y el desarrollo de cardiopatía isquémica. La adrenalina potencia las hemostasias primaria y secundaria - puede producir trombosis intracoronaria- y causa vasoconstricción transitoria arteriolar. Se presenta un caso clínico de una paciente que acudió a urgencias por dolor torácico pocas horas después de una intervención dentaria. En el estudio posterior se descubrió un infarto de miocardio por trombosis intracoronaria de la descendente anterior distal.

(c) 2020 Sociedad Colombiana de Cardiología y Cirugía Cardiovascular. Publicado por Elsevier España, S.L.U. Este es un artículo Open Access bajo la licencia CC BY-NC-ND (http:// creativecommons.org/licenses/by-nc-nd/4.0/).

Myocardial infarction after intra-gingival administration of adrenaline. A new cause of myocardial infarction without obstructive coronary atherosclerosis?

\begin{abstract}
There is a relationship between adrenaline administered intramuscularly and the development of ischaemic heart disease. Adrenaline promotes primary and secondary haemostasis, which can produce a coronary thrombosis and cause a transient arteriolar vasoconstriction. A case is presented of a patient who was seen in the Emergency Department with chest pain a few hours after dental treatment. In the subsequent work-up, it was discovered that she had a myocardial infarction due to a coronary thrombosis of the left anterior distal artery.

(c) 2020 Sociedad Colombiana de Cardiología y Cirugía Cardiovascular. Published by Elsevier España, S.L.U. This is an open access article under the CC BY-NC-ND license (http:// creativecommons.org/licenses/by-nc-nd/4.0/).
\end{abstract}

* Autor para correspondencia.

Correo electrónico: pedroperezdiaz61@gmail.com (P. Pérez Díaz). 


\section{Introducción}

La adrenalina (epinefrina) puede constituir en ocasiones un factor precipitante de infarto de miocardio en ausencia de lesiones angiográficamente significativas ${ }^{1-9}$. Este fármaco, a nivel plaquetario, induce una activación de la hemostasia primaria y secundaria. En la piel y las encías estimula los receptores alfa 1-adrenérgicos, produciendo vasoconstricción de las arterias pequeñas y arteriolas. La anestesia permanece más tiempo en el área inyectada, por lo que su efecto es más largo y las concentraciones que llegan a los distintos órganos son menores ${ }^{10,11}$. En el corazón la adrenalina produce aumento de la precarga, el gasto cardíaco y la presión arterial sistólica (PAS). En los vasos sanguíneos, a dosis bajas, estimula los receptores beta 2-adrenérgicos y produce vasodilatación arterial. Por su parte, a dosis medias y altas, incide sobre los receptores alfa 1 -adrenérgicos, produciendo vasoconstricción arterial, con el consiguiente aumento de la presión arterial diastólica (PAD) ${ }^{10}$. A dosis altas, y con la administración intravascular inadvertida, puede producir ángor de reposo, palpitaciones, diaforesis, ansiedad, irritabilidad, nerviosismo e hipertensión arterial ${ }^{11}$. Sin embargo, es su utilización por vía intramuscular o endovenosa como tratamiento de una reacción anafiláctica la que con mayor frecuencia se relaciona con el infarto agudo de miocardio sin enfermedad coronaria aterosclerótica obstructiva ${ }^{12-15}$.

\section{Caso}

Mujer de 71 años de edad, con antecedentes personales de esclerosis aórtica y cardiopatía hipertensiva, quien acudió al servicio de urgencias por dolor centrotorácico opresivo, no irradiado ni asociado a cortejo vegetativo, de tres horas de duración. Como dato de interés comentó que había acudido al dentista horas antes y que había sido sometida a una endodoncia.

Se registró frecuencia cardíaca de $78 \mathrm{lpm}$, tensión arterial de $124 / 74 \mathrm{~mm} \mathrm{Hg}$ y saturación de oxígeno del $95 \%$ basal. La auscultación cardiopulmonar era rítmica y sin soplos, con estertores subcrepitantes de campos pulmonares medios e inferiores. La exploración abdominal reveló un abdomen blando y depresible, sin dolor a la palpación superficial ni profunda, sin peritonismo. Las extremidades inferiores no mostraban edemas maleolares, con pulsos pedios y tibiales posteriores presentes y simétricos.

Los biomarcadores cardíacos fueron CPK $1127 \mathrm{UI} / \mathrm{l}$ pico, CK-MB 119,11 ng/ml pico y troponina-I 29,130 ng/ml pico. La radiografía anteroposterior de tórax portátil mostró índice cardiotorácico conservado, con redistribución vascular, aumento de la trama broncovascular bilateral e infiltrados intersticiales bilaterales y difusos. El electrocardiograma reveló ritmo sinusal a $60 \mathrm{lpm}, \mathrm{PR} 180 \mathrm{~ms}$, QRS estrecho, eje del QRS a $30^{\circ}$, supradesnivelación persistente del segmento ST < $1 \mathrm{~mm}$ en II, III, AVF, V4-V6 y QTc $394 \mathrm{~ms}$ (fig. 1).

Se administró dosis de carga de doble antiagregación y se inició tratamiento médico antianginoso. Dada la persistencia de la clínica y los hallazgos electrocardiográficos se realizó un cateterismo urgente en el que se apreció imagen compatible con trombosis de la descendente anterior,

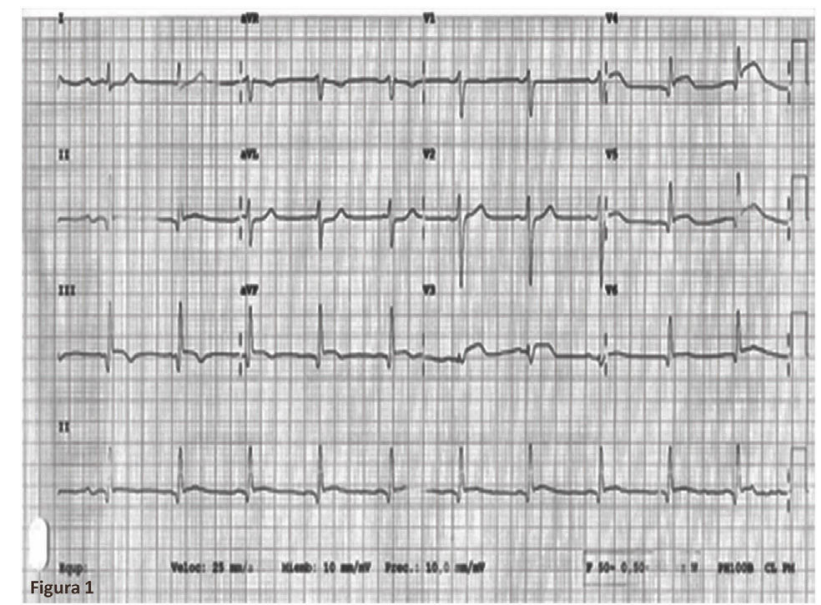

Figura 1 Electrocardiograma. Ascenso del segmento ST en cara inferolateral.

muy distal, siendo este un vaso muy largo, que daba la vuelta al ápex (fig. 2). La ventriculografía mostró hipoquinesia anterior-apical e inferior-apical (vídeo 1). No se realizó tromboaspiración ni angioplastia coronaria debido a la localización distal del trombo; se trató con doble antiagregación y anticoagulación.

Fue trasladada a la unidad coronaria, donde se completó la seriación enzimática y electrocardiográfica. Un ecocardiograma transtorácico mostró ausencia de valvulopatías, aurículas no dilatadas, ventrículo izquierdo no dilatado ni hipertrofiado, sin anomalías groseras de la contractilidad segmentaria, patrón diastólico de alteración de la relajación y función sistólica conservada (FEVI 55\% por Simpson biplano). No se evidenciaron signos de hipertensión pulmonar; el pericardio era de grosor normal, al igual que la refringencia, sin derrame, vena cava inferior no dilatada, con colapso mayor al $50 \%$ con la inspiración (fig. 3).

Durante su estancia en la unidad coronaria permaneció estable, con cifras tensionales con tendencia a la hipotensión y afebril. No presentó nuevos episodios de ángor de reposo/ligeros esfuerzos, palpitaciones ni disnea. Ante la estabilidad clínica y hemodinámica fue dada de alta a planta de Cardiología. Previo al alta la paciente aportó el informe de la extracción dental realizada en un centro privado unas horas antes de la aparición de la clínica, en el que figuraba la utilización de un anestésico local compuesto por lidocaína $2 \%$ más epinefrina $(0,0225 \mathrm{mg}$ $[0,01 \mathrm{mg} / \mathrm{ml}])$.

Posteriormente se realizó cardiorresonancia magnética con gadolinio, la cual reveló aquinesia anteroseptal apical con captación subendocárdica mayor del 50\% a nivel anteroseptal apical y apical estricto, y menor del $50 \%$ en región anterolateral basal, todo ello compatible con infarto agudo de miocardio como primera posibilidad. Así pues, fue dada de alta con recomendaciones higiénico-dietéticas, doble antiagregación, betabloqueadores, inhibidores de la enzima convertidora de angiotensina y estatinas. Un año más tarde permanece clínicamente asintomática y sin alteraciones de la contractilidad segmentaria por ecocardiografía. 


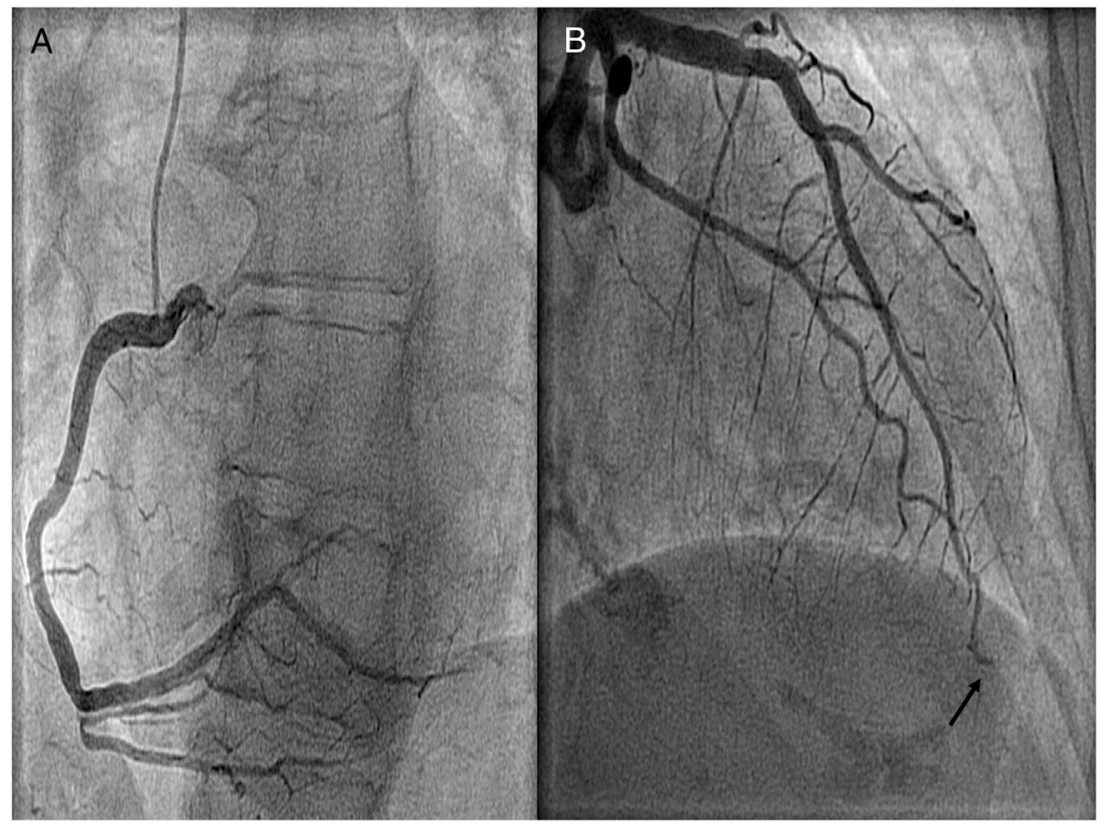

Figura 2 Coronariografía. Trombosis de la descendente anterior a nivel muy distal.

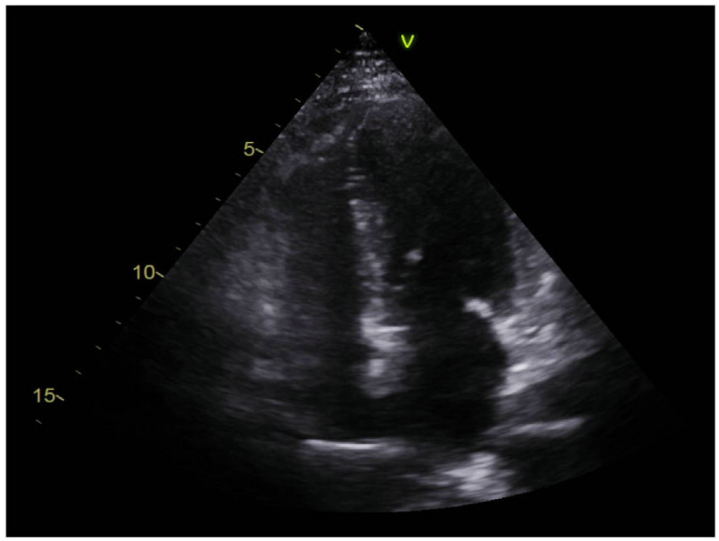

Figura 3 Ecocardiograma transtorácico. Ventrículo izquierdo no dilatado ni hipertrofiado. Ausencia de valvulopatías significativas.

\section{Discusión}

A nivel plaquetario la adrenalina induce un incremento en la síntesis y liberación de tromboxano $A 2$ y B2, así como un aumento de la afinidad de los receptores P2Y1 y P2Y12, fenómenos implicados en la hemostasia primaria. Asimismo, la adrenalina plaquetaria tiene la capacidad de incrementar la actividad de la trombina, enzima que, a su vez, se encarga de la degradación del fibrinógeno en monómeros de fibrina, generando puentes interplaquetarios, acción fundamental en el contexto de la hemostasia secundaria. En el corazón la adrenalina produce efectos cronotrópico, dromotrópico, batmotrópico e inotrópico positivos, y en los vasos sanguíneos, a dosis medias y altas, incide sobre los receptores alfa1-adrenérgicos, produciendo vasoconstricción arterial, con el consiguiente aumento de la presión arterial diastólica.
La relación entre la adrenalina y la cardiopatía isquémica se encuadra casi siempre en el contexto de un síndrome de hipersensibilidad, con la utilización de este fármaco por vía intramuscular o endovenosa. Jackson et al., Kasim et al. y Cunnington et al. describieron sendos reportes clínicos en los cuales el común denominador era la aparición de un infarto de miocardio relacionado con la administración de adrenalina por vía intramuscular como tratamiento de una reacción de hipersensibilidad significativa ${ }^{13-15}$. El primer paciente presentaba antecedentes personales de cardiopatía isquémica con un stent en la arteria descendente anterior hacía cuatro años, y en la imagen angiográfica actual mostraba una trombosis muy tardía del stent. Se realizó tromboaspiración y angioplastia con balón no compliante, con buen resultado angiográfico. En el segundo caso también se evidenció una imagen compatible con trombosis intracoronaria, que se resolvió con tromboaspiración, sin necesidad de angioplastia. En estos pacientes el mecanismo más plausible constituye la potenciación de la hemostasia tanto primaria como secundaria por parte de la adrenalina, tal y como se ha comentado previamente. Sin embargo, puesto que el primer paciente era fumador, tampoco se descarta una acción directa del tabaco sobre el endotelio vascular que resultara en un fenómeno de trombosis sin presencia de placa de ateroma previa. Asimismo, ya que en fase aguda no se realizaron técnicas de imagen intracoronaria, no se pudo descartar la presencia de placa de ateroma extrínseca ni hematoma o disección coronaria. En el último caso, no obstante, la coronariografía preferente objetivó arterias coronarias sin lesiones angiográficamente significativas. En este caso se asumió una vasoconstricción arterial inducida por la adrenalina intramuscular como mecanismo subyacente. Esta hipótesis se hace si cabe mayor al conocer que la paciente se encontraba tomando un betabloqueador no cardioselectivo, como el propranolol, con el consiguiente disbalance vasoconstricción-vasodilatación en presencia de una catecolamina como la adrenalina. 


\section{Conclusiones}

En el caso expuesto la curva enzimática fue compatible con un infarto de miocardio, por lo que se descarta que ese fuese el electrocardiograma basal de la paciente. Tampoco se describieron alteraciones hidroelectrolíticas en los primeros análisis. Un ecocardiograma transtorácico reglado no mostró anomalías groseras de la contractilidad segmentaria, lo cual hacía el diagnóstico de takotsubo poco probable. Además, la cardiorresonancia fue compatible con infarto agudo de miocardio en fase subaguda. La relación entre la adrenalina y la cardiopatía isquémica se encuadra en el contexto de una reacción de hipersensibilidad. Sin embargo, este no era el caso ya que la administración de adrenalina se descubrió "a posteriori", y no se usó como tratamiento de una reacción de hipersensibilidad sino como coadyuvante de anestésico local (a una dosis usual 0,01 $\mathrm{mg} / \mathrm{ml}$ ). La adrenalina pudo producir un fenómeno de trombosis intracoronaria, que se evidenció en la angiografía. Así pues, una vez planteadas todas las hipótesis, se propone que el mecanismo más plausible del cuadro clínico de la paciente fue la trombosis intracoronaria de la descendente anterior debido a una inyección intravascular inadvertida de adrenalina como coadyuvante anestésico. Este sería, por tanto, el primer caso descrito en la literatura médica de infarto de miocardio secundario a inyección de adrenalina intragingival.

\section{Financiación}

Ninguna.

\section{Conflictos de intereses}

Ninguno.

\section{Anexo. Material adicional}

Se puede consultar material adicional a este artículo en su versión electrónica disponible en doi:10.1016/j.rccar. 2019.12.013.

\section{Bibliografía}

1. Niccoli G, Scalone G, Crea F. Acute myocardial infarction with no obstructive coronary atherosclerosis: mechanisms and management. Eur Heart J. 2015;36:475-81.

2. Kubo T, Imanishi T, Takarada S, Kuroi A, Ueno S, Yamano T, et al. Assessment of culprit lesion morphology in acute myocardial infarction: ability of optical coherence tomography compared with intravascular ultrasound and coronary angioscopy. J Am Coll Cardiol. 2007;50:933-9.

3. Takagi Y, Yasuda S, Takahashi J, Tsunoda R, Ogata Y, Seki A, et al. Clinical implications of provocation tests for coronary artery spasm: safety, arrhythmic complications and prognostic impact multicentre registry study of the Japanese Coronary Spasm Association. Eur Heart J. 2013;34:258-67.

4. Wittstein IS, Thiemann DR, Lima JA, Baughman KL, Schulman SP, Gerstenblith G, et al. Neurohumoral features of myocardial stunning due to sudden emotional stress. N Engl J Med. 2005;352:539-48.

5. Donohue D, Movahed MR. Clinical characteristics, demographics and prognosis of transient left ventricular apical ballooning síndrome. Heart Fail Rev. 2005;10:311-6.

6. Prasad A. Apical ballooning syndrome: an important differential diagnosis of acute myocardial infarction. Circulation. 2007;115, e-56-9.

7. Leurent G, Langella B, Fougerou C, Lentz PA, Larralde A, Bedossa $M$, et al. Diagnostic contributions of cardiac magnetic resonance imaging in patients presenting with elevated troponin, acute chest pain síndrome and unobstructed coronary arteries. Arch Cardiovasc Dis. 2011;104:161-70.

8. Shibata T, Kawakami S, Noguchi T, Tanaka T, Asaumi Y, Kanaya $\mathrm{T}$, et al. Prevalence, clinical features and prognosis of acute myocardial infarction attributable to coronary artery embolism. Circulation. 2015;132:241-50.

9. Domínguez F, Kühl U, Pieske B, García-Pavía P, Tschöpe C. Actualización sobre miocarditis y miocardiopatía inflamatoria: el resurgir de la biopsia endomiocárdica. Rev Esp Cardiol. 2016;69:178-87.

10. Muñoz JI, Rosellón P. Protocolo fármacos vasoactivos: dosis, indicaciones y efectos adversos Hospital Clínico Universitario. Servicio de Pediatría. Sección de $\mathrm{Cl}$ y Urgencias Pediátricas. 2013.

11. Wallen NH, Held C, Rehnqvist N, Hjemdahl P. Effects of mental and physical stress on platelet function in patients with stable angina pectoris and healthy controls. Eur Heart J. 1997; 18:807-15.

12. Burgess J, Meyers AD. Dental management in the medically compromised patient Medscape Drugs \& Diseases. Clinical Procedures. 2015. Disponible en: https://emedicine.medscape.com/article/2066164-overview.

13. Jackson CE, Dalzell JR, Hogg KJ. Epinephrine treatment of anaphylaxis: An extraordinary case of very late acute stent thrombosis. Circ Cardiovasc Interv. 2009;2:79-82.

14. Kasim S, AbuBakar R, McFadden E. Anaphylaxis from wasp stings inducind coronary thrombus. Case Rep Cardiol. 2012;2012:701-53

15. Cunnington C, McDonald JE, Singh RK. Epinephrine-induced myocardial infarction in severe anaphylaxis: is nonselective B-blockade a contributory factor? Am J Emerg Med. 2013; 31:759. 